\title{
Greg Restall Normal Proofs, Cut Free Derivations and Structural Rules
}

\begin{abstract}
Different natural deduction proof systems for intuitionistic and classical logic - and related logical systems - differ in fundamental properties while sharing significant family resemblances. These differences become quite stark when it comes to the structural rules of contraction and weakening. In this paper, I show how Gentzen and Jaśkowski's natural deduction systems differ in fine structure. I also motivate directed proof nets as another natural deduction system which shares some of the design features of Genzen and Jaśkowski's systems, but which differs again in its treatment of the structural rules, and has a range of virtues absent from traditional natural deduction systems.
\end{abstract}

Keywords: Natural deduction, Normal proof, Sequent calculus, Structural rule, Proof net.

\section{Representing proof: Jaśkowski, Gentzen and Beyond}

Logic is about many different things. One of the things logic is about is the notion of proof. A traditional natural deduction proof displays inferential connections in a deduction from some set (maybe an empty set) of premises to some conclusion. A clear and precise conception of proof gives you the means to see how those inferential connections arise from some small collection of fundamental inferential steps, in a manner not too dissimilar from how the variety of molecules are made up from the atoms on the periodic table.

The 1930s was a time of flowering for our understanding of proof, especially in the work of logicians like Jaśkowski [12] and Gentzen [8], who independently in the year 1934 wrote on natural deduction proof systems. ${ }^{1}$ Each natural deduction system has at its heart a small number of rules for connectives. The rules come in a particular form, dictated by the shapes of proofs. In the case of Gentzen's natural deduction system, a proof is a tree, and the two rules for the conditional $-\langle\rightarrow E\rangle$ and $\langle\rightarrow I\rangle$ - show how one can construct and extend proof trees in a controlled fashion:

\footnotetext{
${ }^{1}$ Jeff Pelletier gives us an excellent historical account of the early development of natural deduction [21].

Special Issue: Gentzen's and Jaśkowski's Heritage 80 Years of Natural Deduction and Sequent Calculi Edited by Andrzej Indrzejczak
} 


$$
\frac{A \rightarrow B \quad A}{B \quad\langle\rightarrow E\rangle} \quad \begin{gathered}
{[A]^{(i)}} \\
\frac{\dot{B}}{A \rightarrow B}\left\langle\rightarrow I_{i}\right\rangle
\end{gathered}
$$

The $\langle\rightarrow E\rangle$ rule is not only a small proof in its own right. We also conventionally read this rule (and all rules of this general form) as saying that if we have one proof, $\pi_{1}$, ending in $A \rightarrow B$, and another, $\pi_{2}$, ending in $A$, then the tree consisting of $\pi_{1}$ in the left branch, $\pi_{2}$ in the right branch, and $B$ at the root (the conclusion) is itself a proof. This proof has, as premises, the premises of $\pi_{1}$ together with the premises of $\pi_{2}$.

The $\langle\rightarrow I\rangle$ rule is slightly more complex. It tells us that if we already have a proof with $B$ as a conclusion (indicated by the vertical ellipsis leading to $B$ ) then we can extend this proof to the conclusion $A \rightarrow B$ while discharging as many premises of the form $A$ in that proof as we wish. ('As many as we wish' can include zero. This will become important in what follows.) We indicate this in a proof by enclosing the discharged assumptions in square brackets, and (optionally) tagging them with a numeral (here it is $i$ ) which pairs the discharged formulas with this particular instance of the rule. That pairing is important when instances of the one formula are discharged at different points in the proof. Here is a proof, displaying some of the distinctive features of Gentzen's system. The proof leads from the premise $p \rightarrow r$ to the conclusion $(p \rightarrow(r \rightarrow s)) \rightarrow(p \rightarrow(q \rightarrow s))$. All other leaves in the proof are assumptions discharged at different stages of the proof.

$$
\begin{gathered}
\frac{[p \rightarrow(r \rightarrow s)]^{(3)}[p]^{(2)}\langle\rightarrow E\rangle \quad \frac{p \rightarrow r \quad[p]^{(2)}}{r \rightarrow s}\langle\rightarrow E\rangle}{\frac{s}{q \rightarrow s}\left\langle\rightarrow I_{1}\right\rangle}\langle\rightarrow E\rangle \\
\frac{\frac{\rho}{p \rightarrow(q \rightarrow s)}\left\langle\rightarrow I_{2}\right\rangle}{(p \rightarrow(r \rightarrow s)) \rightarrow(p \rightarrow(q \rightarrow s))}\left\langle\rightarrow I_{3}\right\rangle
\end{gathered}
$$

You may notice that the discharge at step $\left\langle\rightarrow I_{1}\right\rangle$ is what we might describe as vacuous. We have discharged an empty set of formula instances. There is no assumption $q$ tagged with a 1 in the leaves of the proof. (In fact, $q$ is not used as an assumption in the proof.) On the other hand, the assumption $p$ discharged at step $\left\langle\rightarrow I_{2}\right\rangle$ is discharged twice, once in the left subproof and once in the right. 
Here is another proof, which shows some of the ways we can take in indirect route to prove a conclusion.

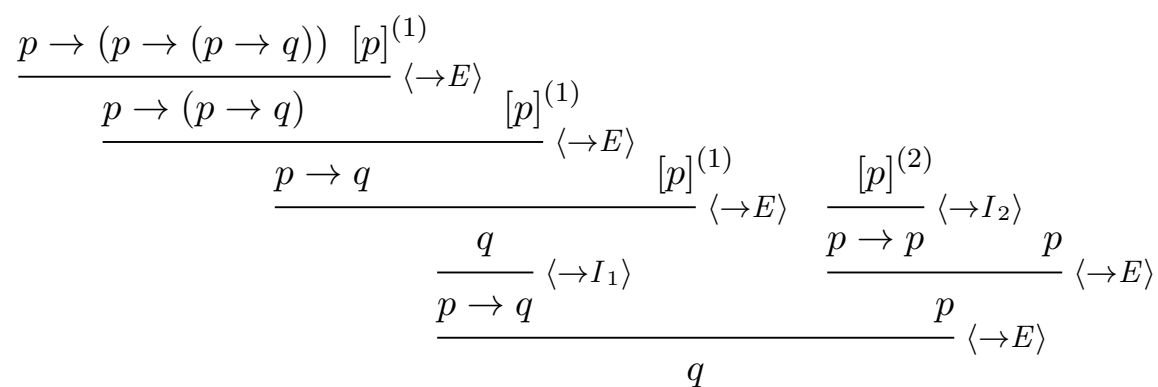

This proof leads us from the premises $p \rightarrow(p \rightarrow(p \rightarrow q))$ (the leftmost leaf of the tree) and $p$ (the rightmost) to the conclusion $q$. However, it does this in a circuitous way: the conclusion $q$ is reached after the second $\langle\rightarrow E\rangle$ step in the left branch of the tree, but after this, a conditional is introduced only to be eliminated again. This introduction followed by an elimination is redundant, and the proof tree can be simplified, by taking the two places that $p$ is discharged (tagged with 1 , since these are the instances of $p$ discharged in the $\left\langle\rightarrow I_{1}\right\rangle$ step), and justifying these using the proof of the minor premise $p$ in the right branch of the tree.

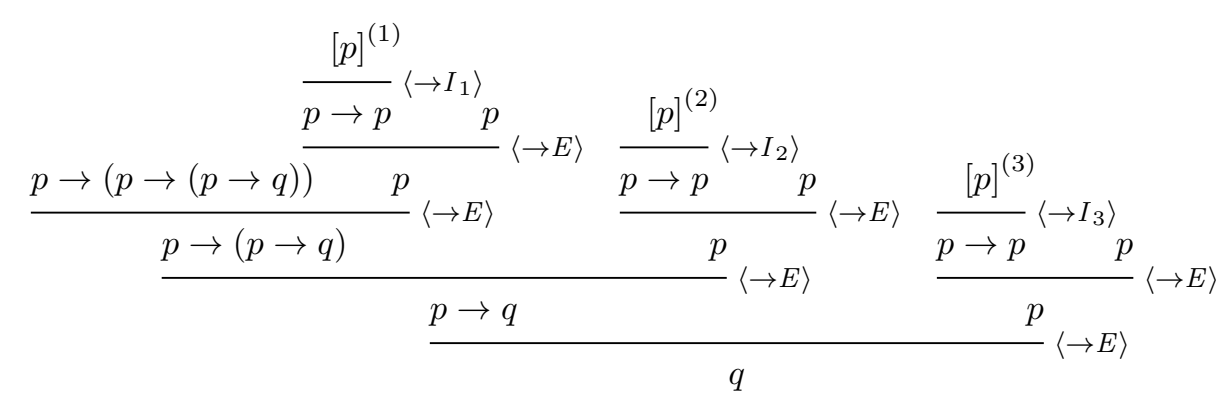

You can see that this proof is 'simpler' in that it doesn't contain that redundancy. However, the original proof contains 13 formulas, while this 'simpler' proof is larger, containing 16 formulas. The subproof justifying $p$ is reproduced three times in place of the three instances of the assumption $p$ discharged at step $\left\langle\rightarrow I_{1}\right\rangle$ in the original proof. (Note that the assumption numbering in the subproof is changed as we subtitute that subproof into the three different locations. I won't go into the detail of how this can be done systematically. The details of what numbers are used where is not important for the identity or difference of two distinct presentations of a proof.)

However, even though we have simplified a redundancy in the original proof, this new proof contains three more redundancies, where a conditional 
is introduced and then eliminated. The same redundant derivation of $p$ from $p$ (using the major premise $p \rightarrow p$ ) occurs in each of the subproofs. We can eliminate each these detours. The result is a much simpler proof, which contains only 7 formulas.

$$
\frac{p \rightarrow(p \rightarrow(p \rightarrow q)) \quad p}{\left.\frac{p \rightarrow(p \rightarrow q)}{p \rightarrow q} p\right\rangle} \frac{p}{q}\langle\rightarrow E\rangle{ }^{p}\langle\rightarrow E\rangle
$$

Although this proof is made out of only 7 formulas, it has three separate instances of the undischarged assumption $p$, while the original proof used only one instance of that assumption.

A natural deduction proof without such detours is said to be normal, and procedures for transforming a non-normal proof to a normal one is called, as you might expect, normalisation. A system has a normalisation theorem if it can be shown that each non-normal proof can be transformed in some way into a normal proof [23].

Gentzen's technique for natural deduction is not the only way to represent this kind of reasoning, with introduction and elimination rules for connectives. Independently of Gentzen, Stanisław Jaśkowski constructed a distinct system for presenting proofs in a natural deduction style. In Jaśkowski's system, a proof is a structured list of formulas, rather than a tree. Each formula in the list is either a supposition, or it follows from earlier formulas in the list by means of the rule of modus ponens (conditional elimination), or it is proved by conditionalisation. To prove something by conditionalisation you first make a supposition of the antecedent: at this point you start a box. The contents of a box constitute a proof, so if you want to use a formula from outside the box, you may repeat a formula inside that box so it is available for use. A conditionalisation step allows you to exit the box, discharging the supposition you made upon entry. Boxes can be nested, as follows: 


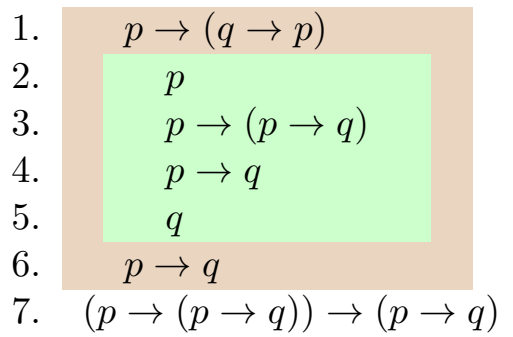

Supposition
Supposition
Supposition
2, 3, Modus Ponens
2, 4, Modus Ponens
1, Repeat
1-6, Conditionalisation

This nesting of boxes, and repeating or reiteration of formulas to enter boxes, is the distinctive feature of Jaśkowski's system. ${ }^{2}$

Notice that in this proof we concluded the formula $(p \rightarrow(p \rightarrow q)) \rightarrow$ $(p \rightarrow q)$ without using a duplicate discharge. We did not need to make the assumption $p$ twice. The formula $p$ is used twice as a minor premise in a Modus Ponens step (on line 4, and on line 5 respectively), and it is then discharged once at line 6 . In a normal Gentzen proof of the same formula, the assumption $p$ would have been be made twice.

Jaśkowski proofs also straightforwardly incorporate the effects of a vacuous discharge in a Gentzen proof. We can prove $p \rightarrow(q \rightarrow p)$ using the rules as they stand, without making any special plea for a vacuous discharge:

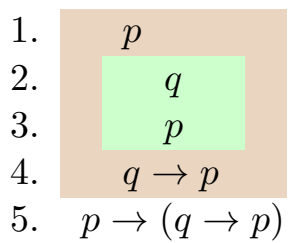

Supposition

Supposition

1, Repeat

2-3, Conditionalisation

1-4, Conditionalisation

The formula $q$ is supposed, and it is not used as a premise to derive anything else in the proof that follows. Instead, it is discharged immediately after the innocent bystaner $p$ is brought into its scope. The formula $p$ on line 3 occurs after the formula $q$ on line 3 , in the subproof, but it is hard to see how it might have been inferred from that $q$. Conditionalisation, in Jaśkowski's system, colludes with reiteration to allow the effect of vacuous discharge.

With that introduction to Jaśkowski-style natural deduction, we can see how to use this system to present the same kind of reasoning as in the Gentzen proof from $p \rightarrow r$ to $(p \rightarrow(r \rightarrow s)) \rightarrow(p \rightarrow(q \rightarrow s))$ given on page 2 .

\footnotetext{
${ }^{2}$ These are features shared by Fitch-style natural deduction [7]. Natural deduction in the style of Suppes [32] or Lemmon [15] is linear, but does not require repeating or reiteration of formulas because assumptions are encoded by numbering rather than by the structural feature of the nesting of boxes.
} 


\begin{tabular}{r|c}
1. & $p \rightarrow r$ \\
2. & $p \rightarrow(r \rightarrow s)$ \\
3. & $p$ \\
4. & $p \rightarrow(r \rightarrow s)$ \\
5. & $r \rightarrow s$ \\
6. & $p \rightarrow r$ \\
7. & $r$ \\
8. & $s$ \\
9. & $q$ \\
10. & $\quad s \rightarrow s$ \\
11. & $p \rightarrow(q \rightarrow s)$ \\
12. & $(p \rightarrow(r \rightarrow s)) \rightarrow(p \rightarrow(q \rightarrow s))$ \\
13. &
\end{tabular}

\author{
Supposition \\ Supposition \\ Supposition \\ 2, Repeat \\ 3,4, Modus Ponens \\ 1, Repeat \\ 3,6 Modus Ponens \\ 5,7, Modus Ponens \\ Supposition \\ 8, Repeat \\ 9-10, Conditionalisation \\ 3-11, Conditionalisation \\ 2-12, Conditionalisation
}

This is not an exact match of the Gentzen proof of page 2, but it comes close. The Jaśkowski proof has matching Modus Ponens steps for every $\langle\rightarrow E\rangle$ step in the Gentzen proof, and Conditionalisation steps for every $\langle\rightarrow I\rangle$. However, the two short subproofs, one of $r \rightarrow s$ in the left branch, and the other, of $r$ in the right branch, are totally independent of one another in the Gentzen proof. The undischarged assumption of $p \rightarrow r$ in the right subproof is not inside the scope of the discharged assumption of $p \rightarrow(r \rightarrow s)$ in the left subproof, and neither is $p \rightarrow(r \rightarrow s)$ within the scope of the assumption of $p \rightarrow r$. They are logically independent of one another. This cannot be said of the Jaśkowski proof. Since it is linear, a choice must be made as to the order in which the formulas occur. The assumption $p \rightarrow r$ governs the entire proof, and here, the proof of $r \rightarrow s$ from $p \rightarrow(r \rightarrow s)$ and $p$ (lines $3-5)$ occurs inside the scope region of the assumption of $p \rightarrow r$.

There is a certain degree of "fine control" over inferential connections between formulas in a Gentzen proof. Some of these finer distinctions are obscured in the linearisation of a Jaśkowski proof. This is the price paid for having a linear proof structure. The fact that one formula occurs after another does not mean that must be inferentially connected to the formulas that came before it. In proofs, as in life, your elders need not be your ancestors.

Despite the differences, there are significant similarities between Gentzen's and Jaśkowski's treatment of natural deduction. Connectives have introduction and elimination rules, and in Jaśkowski-style proofs just as in Gentzen-style proofs, we can have non-normal proofs in which an introduction is immediately followed by an elimination, and we could define normalisation in a similar way. However, normalisation proceeds in a different 
way in the presence of linear proofs. Consider the obvious Jaśkowski-style analogue to the non-normal Gentzen proof we considered on page 3.

\begin{tabular}{r|c}
1. & $p \rightarrow(p \rightarrow(p \rightarrow q))$ \\
2. & $p$ \\
3. & $p \rightarrow(p \rightarrow(p \rightarrow q))$ \\
4. & $p \rightarrow(p \rightarrow q)$ \\
5. & $p \rightarrow q$ \\
6. & $q$ \\
7. & $p \rightarrow q$ \\
8. & $p$ \\
9. & $p$ \\
10. & $p \rightarrow p$ \\
11. & $p$ \\
12. & $p \rightarrow q$ \\
13. & $q$
\end{tabular}

Supposition
Supposition
1, Repeat
2,3, Modus Ponens
2,4, Modus Ponens
2,5, Modus Ponens
2-6, Conditionalisation
Supposition
Supposition
9-9, Conditionalisation
8,10, Modus Ponens
7, Repeat
11,12, Modus Ponens

This Jaśkowski proof is a proof of the conclusion $q$ within the scope of two suppositions: $p \rightarrow(p \rightarrow(p \rightarrow q))$ (line 1) and $p$ (line 8). A Modus Ponens logically follows a Conditionalisation step in the inner subproof (lines 10 and 11 ) and in the main proof (the conditional introduced at 7 is next used at 13 , via its repetition at line 12 , in a modus ponens), so there are two different spots to normalise this proof. To normalise, we eliminate both redundant pairs and we end up with the following simpler proof:

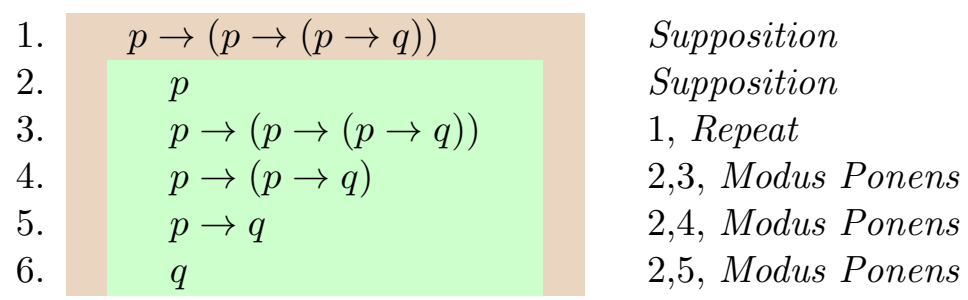

This is a proof of $q$ from the same assumptions, $p \rightarrow(p \rightarrow(p \rightarrow q)$ ) (line 1 ) and $p$ (this time on line 2). Notice that this proof differs significantly from the normal Gentzen proof on page 4, since the assumption $p$ occurs here only once, rather than occuring 3 times. This is because discharing is very different in Jaśkowski proofs. These have no direct analogue of duplicate discharge in Gentzen proofs. Only one instance of a formula is discharged at any one time. However, a discharged formula may be used repeatedly, as the assumption on line 2 is in this proof: it is the minor premise for the 
Modus Ponens steps on lines 4, 5 and 6, corresponding to the three $\langle\rightarrow E\rangle$ steps constituting the Gentzen proof.

That is one difference between Gentzen proofs and Jaśkowski proofs, centred on duplicate discharge and the structural rule of contraction. There is another significant difference in the structure of proofs, on how vacuous discharge and the structural rule of weakening is treated. Here is a very strange small Jaśkowski proof.

\begin{tabular}{l|lll} 
1. & $A$ & Supposition \\
2. & $B$ & Supposition \\
3. & $A$ & 1, Repeat
\end{tabular}

This proof has no direct analogue in a Gentzen system. We can, of course, construct a proof from $A$ and $B$ to $A$ in any number of ways. If we had the standard Gentzen rules for conjunction, we could reason as follows.

$$
\frac{A \quad B}{\frac{A \wedge B}{A}\langle\wedge I\rangle}\langle\wedge\rangle
$$

The result is a proof with $A$ and $B$ as premises and $A$ as the conclusion. But we need not use conjunction to turn this trick - the rules for the conditional will do just as well:

$$
\frac{\frac{A}{B \rightarrow A}\left\langle\rightarrow I_{1}\right\rangle}{A}\langle\rightarrow E\rangle
$$

Here we use the vacuous discharge in the $\langle\rightarrow I\rangle$ step to bring $B$ into the proof. $B$ isn't really used in the proof of $A$, but at the very least, this proof allows us to bring $B$ alongside $A$. These proofs both take the circuitous step through a fresh connective to achieve this result. That's by necessity, in the Gentzen system. Both of these proofs are not normal, and if we use the usual means to normalise Gentzen proofs, the result is the much simpler proof

\section{$A$}

with premise and conclusion $A$, but it does not explicitly record the distinct formula $B$ as a second premise. This is another significant difference between the two different proof systems. In Gentzen's system for intuitionistic propositional logic, there is no normal proof with the two premises $p$ and 
$q$ (atomic formulas) and with the conclusion $p .^{3}$ It is easy to see why: all normal proofs have the subformula property. A proof has the subformula property if and only if all formulas in that proof are subformulas in the premises and conclusions of the proof. So, if $p$ and $q$ are atomic formulas, and a proof from $p$ and $q$ to $p$ has the subformula property, it follows that $p$ and $q$ are identical. After all, any proof from atoms to atoms with the subformula property cannot contain any formulas with connectives or quantifiers or other complexity (no complex formula is a subformula of an atom), and given that every inference rule in a Gentzen system either introduces or eliminates a complex formula, the proof must contain no inference steps. So, normal proofs from $p$ and $q$ to $p$ (if there are any) must be identity proofs, in which the premise is identical to the conclusion. If it is a proof of $p$, then it must contain $p$ as the only premise. It does not involve $q$ as a distinct extra side premise.

It follows that Jaśkowski's system and Gentzen's systems are not merely notational variants of one another, at least if we wish to respect the fine structure of proofs. Jaśkowski's system has a normal proof from $p$ and $q$ to $p$ while Gentzen's doesn't. Jaśkowski also provides us with a short normal proof from $p \rightarrow(p \rightarrow(p \rightarrow q))$ and $p$ to $q$ in which the assumption $p$ is made only once, and not three times. (However, it is applied three times, not just once.) There are no such normal Gentzen proofs.

Are these distinctions worth recording? Your answer to this will depend on what you're taking the aim of proof theory to be. If proofs are merely a device for telling you whether or not an argument is valid, then there is no distinction worth recording between Jaśkowski and Gentzen systems. The exact same arguments are valid according to both systems. If we are interested not only in provability but in the properties of particular proofs, then these differences may matter. As I've characterised proof theory - as giving an account of the inferential connections between formulas in proofsthen these differences are squarely inside the purview of proof theory.

However, proof theory as studied in the 1930s and into the middle of the 20th Century didn't take the different representations of proofs and their properties as a focus of investigation. Instead, results such as normalisation, cut elimination, and other metatheoretical properties of systems became the focus. The greatest work in this tradition was due to Gerhard Gentzen. His most significant results used not natural deduction, but another way of

\footnotetext{
${ }^{3}$ I use atomic formulas rather than the schematic formula letters $A$ and $B$ to state this result because of course for particular formulas $A$ and $B$ there will be a Gentzen proof which leads from $A$ and $B$ to $A$. (From $p$ and $p \rightarrow p$ to $p$, as but ones example.) There is no proof in the case where $A$ and $B$ are distinct atoms.
} 
representing simple inferential connections: the sequent calculus, which will be our focus in the next section.

\section{Reasoning about proofs: sequent calculus}

In giving an account of the process of normalisation of natural deduction proofs, Gentzen [8] discovered that it makes sense to manipulate sequents. If we have a proof from premises $X$ to conclusion $A$, then the logical content can be recorded as a sequent $X \vdash A$. Gentzen's rules $\langle\rightarrow I\rangle$ and $\langle\rightarrow E\rangle$ can be understood as describing sequents. For $\langle\rightarrow I\rangle$ if we have a proof from $X, A$ to $B$, then if we extend this with an $\langle\rightarrow I\rangle$ step the result is a proof from $X$ to $A \rightarrow B$. We can represent this as a sequent rule:

$$
\frac{X, A \vdash B}{X \vdash A \rightarrow B}\langle\rightarrow R\rangle
$$

We can do the same for $\langle\rightarrow E\rangle$. As we have conventionally read $\langle\rightarrow E\rangle$, It tells us that if we have a proof from $X$ to $A \rightarrow B$ and another proof from $Y$ to $A$ then we can use these to construct a proof from $X, Y$ to $B$. We would have the following inference rule:

$$
\frac{X \vdash A \rightarrow B \quad Y \vdash A}{X, Y \vdash B}
$$

Gentzen's sequent calculus in its modern form does not involve rules like this, ${ }^{4}$ in which a formula $A \rightarrow B$ is eliminated from the premise of the rule to the conclusion. He showed that elimination rules like $\langle\rightarrow E\rangle$ correspond to rules in which the formula $A \rightarrow B$ is introduced on the left of the sequent. Conceive of $\langle\rightarrow E\rangle$ in the following way:

$$
\frac{\stackrel{A \rightarrow B}{{ }^{A}}{ }^{B}}{\dot{ }_{C} \pi^{\prime}}\left\langle\rightarrow E^{\prime}\right\rangle
$$

\footnotetext{
${ }^{4}$ Although Gentzen eliminated elimination rules like these in favour of antecedent introduction rules, but in his paper from 1936 [9] he used rules such as these. As a result,, the system from this paper was the ancestor of Lemmon's and Suppes' style natural deduction, except that Gentzen did not use numbers and formulas but sequents, and preferred trees as representation of proofs over lists.
} 
This tells us that if we have $\pi$, a proof, say, from $X$ to $A$, and a proof $\pi^{\prime}$ from $Y, B$ to $C$ then we can combine these, in order to construct a proof from $X, A \rightarrow B, Y$ to the conclusion $C$, by combining the proofs together and adding the premise $A \rightarrow B$. This motivates Gentzen's sequent rule $\langle\rightarrow L\rangle$.

$$
\frac{X \vdash A \quad Y, B \vdash C}{X, A \rightarrow B, Y \vdash C}\langle\rightarrow L\rangle
$$

Notice that if we use $\langle\rightarrow E\rangle$ in this form, (in which the major premise $A \rightarrow B$ is always an assumption) and $\langle\rightarrow I\rangle$ in the usual way, the resulting Gentzenstyle natural deduction proofs are normal. Proofs constructed out of $\left\langle\rightarrow E^{\prime}\right\rangle$ and $\langle\rightarrow I\rangle$ have the subformula property and are normal by design. ${ }^{5}$ This is reflected in Gentzen sequent derivations constructed out of $\rightarrow L$ and $\rightarrow R$. These also satisfy the subformula property. To construct the analogue of non-normal proofs - sequent derivations which do not satisfy the subformula property, Gentzen introduced the $\langle C u t\rangle$ rule.

$$
\frac{X \vdash A \quad Y, A \vdash B}{X, Y \vdash B}\langle C u t\rangle
$$

This rule is the sequent calculus analogue of composing proofs. If we have a proof from $X$ to $A$ and a proof from $Y$ and $A$ to $B$, if we replace the assmumption of $A$ used in that second proof by the proof from $X$ to $A$ the result is a proof from $Y$ and $X$ together to $B{ }^{6}$

We have said nothing about how vacuous and duplicate dischage is to be understood in a sequent system like this. One aspect of Gentzen's great genius was his decision to not incorporate the effects of discharge into the $\rightarrow R$ rule. If we did, we would have something like this:

$$
\frac{X-A \vdash B}{X \vdash A \rightarrow B}\left\langle\rightarrow R^{\prime}\right\rangle
$$

where $X-A$ is the same premise collection as $X$ except for having zero or more instances of $A$ deleted. This would be a good fit for the natural deduction rules of the Gentzen system. However, this would incorporate the effects of vacuous and duplicate discharge into the rules for a particular

\footnotetext{
${ }^{5}$ Such a natural deduction calculus (with elimination rules always using assumptions) which easily leads to normalization has been provided by Jan von Plato [19, 22].

${ }^{6}$ Note that we have implicitly assumed that the order of premises does not matter in natural deduction proofs. This corresponds to thinking of the premises in a sequent as a multiset or a set but not a list in which the order of items matters.
} 
connective, here the conditional. But as we have seen, the behaviour of the conditional has consequences in the vocabulary without the conditional. We have a non-normal proof from the premises $p$ and $q$ to the conclusion $p$, but any normal proof will lose one premise and become a proof of a single premise. While Jaśkowski systems allow for such a proof, it would be a gain in our understanding to be able to give an account of this sort of proof without the need to bring in one or other connective when it seems to play no essential role in the derivation. Gentzen's genius was to treat these matters as totally independent of the rules for the connectives. He introduced the structural rules, in this case, weakening $\langle K\rangle$ and contraction $\langle W\rangle$.

$$
\frac{X \vdash A}{X, B \vdash A}\langle K\rangle \quad \frac{X, B, B \vdash A}{X, B \vdash A}\langle W\rangle
$$

Now we can be precise and read our $\rightarrow R$ rule as discharging one and only one instance of the antecedent formula in the left hand side of the sequent, and then we can use the structural rules of weakening and contraction to reduce multiple premises to one, ready for discharge, or use weakening to generate a premise for discharge when none was there previously.

The motivation, using natural deduction proofs to inspire sequent derivations, can be used to define a mapping from derivations to proofs. We can think of a sequent derivation as a way of constructing a natural deduction proof. If we consider this very simple example of a Gentzen proof,

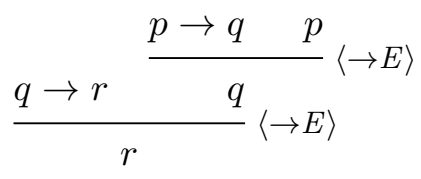

we can think of it as being built up in a number of different ways. These different modes of construction correspond to different sequent derivations. The following two derivations both can be seen as constructing the one and the same proof:

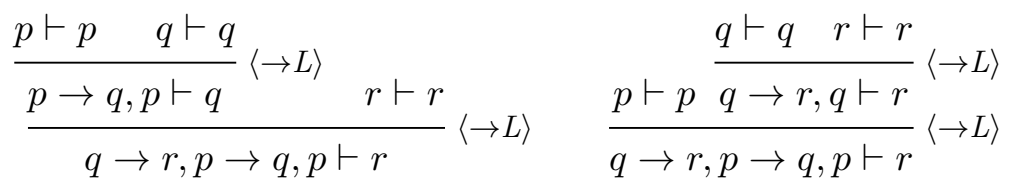

The left derivation focuses on the subproof from $p \rightarrow q$ and $p$ to $q$ and then extends this to $r$ using $q \rightarrow r$ as an extra premise. The right derivation focuses on the subproof from $q$ and $q \rightarrow r$ to $r$ and then extends this by justifying the premise $q$ with two premises $p \rightarrow q$ and $p$. Both are acceptable 
'readings' of the original proof. In fact, there is yet another way of presenting the proof:

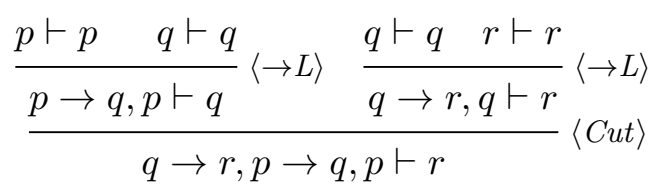

where the intermediate step $q$ is the focus of the $\langle C u t\rangle$ step, chaining together the two subproofs. So, the mapping from sequent calculus derivations to Gentzen-style natural deduction proofs is many-to-one.

Once Gentzen introduced his sequent system, he noticed that a very small modification allowed for the smooth representation of classical propositional logic as well as intuitionistic propositional logic. Once we allow for multiple formulas on the right hand side of the sequent, and allow for the corresponding structural rules of weakening and contraction on the right hand side as well as the left, classical logic straightforwardly follows. The rules now look like this:

$$
\begin{gathered}
\frac{X \vdash A, W \quad Y, B \vdash Z}{X, A \rightarrow B, Y \vdash W, Z}\langle\rightarrow L\rangle \quad \frac{X, A \vdash B, Y}{X \vdash A \rightarrow B, Y}\langle\rightarrow R\rangle \\
\frac{X \vdash Y}{X, B \vdash Y}\langle K\rangle \quad \frac{X \vdash Y}{X \vdash B, Y}\langle K\rangle \quad \frac{X, B, B \vdash Y}{X, B \vdash Y}\langle W\rangle \quad \frac{X \vdash B, B, Y}{X \vdash B, Y}\langle W\rangle
\end{gathered}
$$

The resulting system allows for straightforward (if not exactly intuitive) derivations of distinctively classical sequents, such as that for Peirce's Law.

$$
\begin{gathered}
\frac{p \vdash p}{p \vdash q, p}\langle K\rangle \\
\frac{p \vdash p \frac{1}{(p \rightarrow q \rightarrow q, p}}{(p \rightarrow q) \rightarrow p \vdash p, p}\langle\rightarrow L\rangle \\
\langle W\rangle
\end{gathered}
$$

In this derivation, the relaxation of our structures for sequents to allow for multiple conclusion formulas is necessary to allow for contraction on the right hand side at the last step of the derivation. This makes the interpretation of the sequent calculus less straightforward. If a derivation of a sequent $X \vdash A$ describes the construction of a proof from $X$ to $A$, then what could a sequent derivation of $X \vdash Y$ describe? The many members of $X$ are the many premises of the proof. The members of $Y$ are the many conclusions in a proof? Perhaps sense can be made of this notion, but it would take us too far a field to consider what that story might look like [26]. 
Another consequence of Gentzen's introduction of his sequent calculus is the systematic analysis of how one can transform a derivation involving the $\langle C u t\rangle$ rule into one without it. Gentzen's own papers describe one such strategy, but there are many others in the literature, too. There is a very general technique of Cut elimination due to Curry [4] and generalised by Belnap [3] and others [25], which gives an account of some very general conditions on a proof system sufficient for it to allow for a systematic elimination of the $\langle C u t\rangle$ rule. This has consequences, then, for natural deduction proofs too, for a natural deduction proof constructed by a derivation without $\langle C u t\rangle$ is a normal proof.

\section{Structural rules and what they're good for}

Once we have made structural rules explicit, it is clear that these rules are both useful, and questionable. There is clearly something odd with regard to the structural rule of weakening. This use of weakening is characteristic of the issue.

$$
\frac{\frac{p \vdash p}{p, q \vdash p}}{p \vdash q \rightarrow p}\langle K\rangle
$$

The irrelevant premise $q$ is 'weakened in' to the antecedent of the sequent, and then immediately discharged. If we want the conditional ' $\rightarrow$ ' to record a genuine connection between the antecedent and the consequent, then this move will be rejected. This way lies the Anderson-Belnap tradition of relevance (or relevant) logics $[1,2,6,16]$, as well as other resource sensitive readings [20] used in computer science. The idea has a long history, going back to the early decades of the 20th Century [5].

Similarly, the structural rule of contraction has come in for some sustained examination, both in terms of self-referential paradox such as Curry's paradox [24, 18] but also, vagueness [30, 31]. The structural rule of contraction is most explicitly at issue in the sequent calculus when it comes to using an item as the antecedent conditional twice while only discharging it once, such as in this derivation:

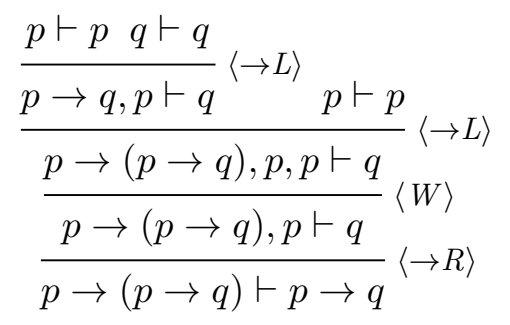


The natural deduction readings of this derivation are less explicit about the form of contraction takes. In a Gentzen system, the deed is done inside the $\langle\rightarrow I\rangle$ step, where two instances of $p$ are discharged at once:

$$
\frac{p \rightarrow(p \rightarrow q)[p]^{(1)}}{\frac{p \rightarrow q}{\frac{q}{p \rightarrow q}}\left\langle\rightarrow I_{1}\right\rangle}\langle\rightarrow]^{(1)}\langle\rightarrow E\rangle
$$

In a Jaśkowski system the trick is turned in a different way:

\begin{tabular}{l|l} 
1. & $p \rightarrow(p \rightarrow q)$ \\
2. & $p$ \\
3. & $p \rightarrow(p \rightarrow q)$ \\
4. & $p \rightarrow q$ \\
5. & $q$ \\
6. & $p \rightarrow q$
\end{tabular}

Supposition

Supposition

1, Repeat

2, 3, Modus Ponens

2, 4, Modus Ponens

2-5, Conditionalisation

As you can see, at line 5, the conclusion $q$ is justified by two uses of the supposition $p$ in the inner subproof.

If we are concerned to develop a natural deduction system for a logic without the rule of contraction, then it is not too difficult to make a Gentzenstyle system: you ban duplicate discharge. If you wish to make a Jaśkowskistyle system, it is more difficult. You cannot ban repetition, since this is necessary for inferences which use no contraction at all. You must ban the repeated use of formulas as premises in modus ponens. This is not straightforward at all.

The mismatch between sequent calculus and natural deduction systems leads us to consider whether there is something like a natural deduction system in which there is a better match with the sequent calculus. Is there a natural deduction system in which classical theorems like Peirce's Law can be proved with a structure paralleling that given in the sequent calculus? Is there a natural deduction system in which structural rules like contraction can be included or omitted at will (hence being more flexible that Jaśkowski systems which essentially incorporate weakening and contraction), and in which weakening and contraction can be available in full generality (so, being more explicit than Gentzen systems, where there is no weakening proof in from $p$ and $q$ to $p$ without involving the connectives). These constraints are satisfied by directed proof nets, or circuit systems. 


\section{Directed proof nets}

Jean-Yves Girard introduced proof nets as a proof syntax designed ideally for multiplicative linear logic, but they are adaptable to a range of other substructural logics $[10,11]$. Proof nets as introduced by Girard are undirected: they correspond to single-sided sequent systems, in which all formulas are conclusions (or, dually, premises). They do not have premises and conclusions. However, it is straightforward to introduce directed proofnets [28, 27], corresponding to full sequents, and the result is a system much closer to traditional natural deduction systems. Directed proofnets are not only motivated by Girard's proofnets, but it also has antecedents in the work of Rudolf Carnap (in his "Tables of Development") and William and Martha Kneale [13, 14], which were then developed in Shoesmith and Smiley's Multiple Conclusion Logic [29]. It is easiest to see the behaviour of proofnets when you consider sequent derivations involving conjunction, disjunction and negation. Proofs are no longer represented with lists or trees of formulas, but as nets, in which nodes are labelled by rules, where arcs are labelled by formulas. The following directed proof net has two inputs $p \vee q$ and $\neg p$ (the premises) and two conclusions $q \wedge \neg r$ and $r$. As with other natural deduction systems, the rules either introduce or eliminate connectives, and the usual sequent derivation can be understood as constructing the proof net.

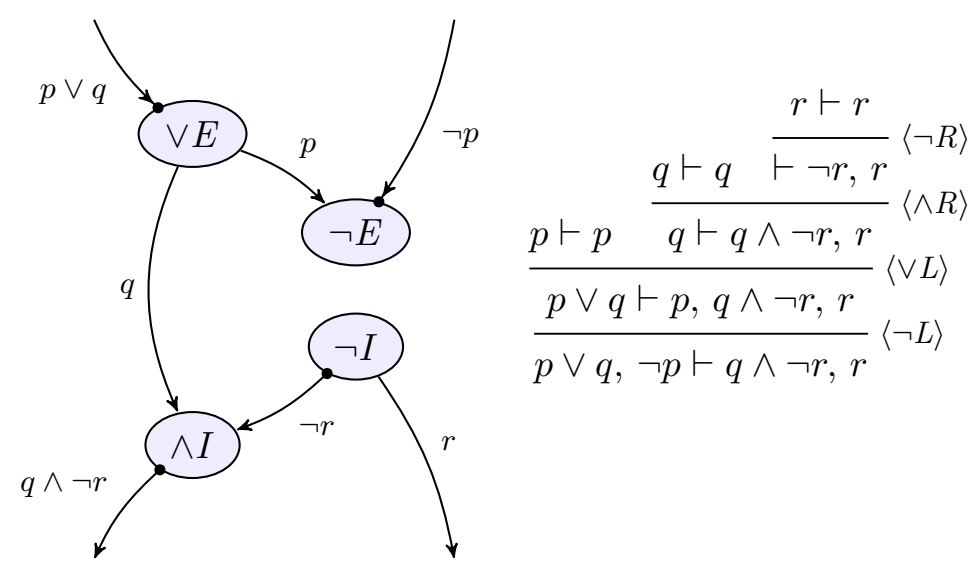

As with other natural deduction systems, the mapping from sequent derivations to proof nets is many-to-one. This different derivation also constructs 
the same proof net.

$$
\frac{\frac{p \vdash p}{p, \neg p \vdash}\langle\neg L\rangle q \vdash q}{\frac{p \vee q, \neg p \vdash q}{p}\langle\vee L\rangle \frac{r \vdash r}{\vdash \neg r, r}}\langle\neg R\rangle
$$

This derivation contains exactly the same rule applications as the first derivation, but it composes them in different order. This order is irrelevant to the directed proof net.

Here is how the sequent calculus derivations describe proof net construction. If we have a proof from $X$ to $Y, A$ this is a circut with inputs $X$ and outputs $Y$ and $A$. This is the box labelled $\pi_{1}$. A proof from $X^{\prime}, B$ to $Y^{\prime}$ is a proof with inputs $X^{\prime}$ and $B$ and outputs $Y^{\prime}$. We compose these with an $\langle\rightarrow E\rangle$ node, which has inputs $A$ and $A \rightarrow B$ (this input marked with a dot - it is the active port on the node) and output $B$. This composes the proof by filling its in its input $A$ with the output $A$ of $\pi_{1}$ and plugging in its output $B$ into the input $B$ required by $\pi_{2}$. The resulting proof has inputs $X, A \rightarrow B, X^{\prime}$ and outputs $Y, Y^{\prime}$, as desired.

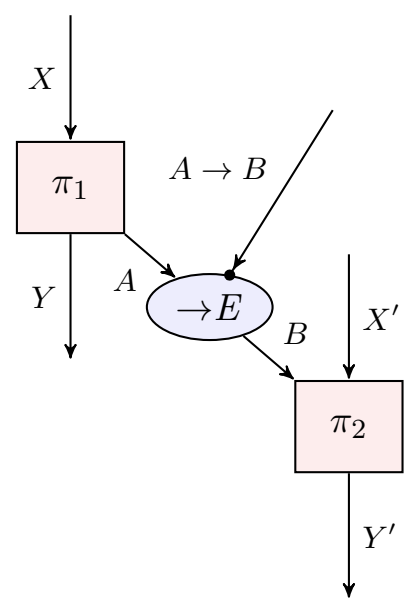

$$
\frac{X \vdash Y, A \quad X^{\prime}, B \vdash Y^{\prime}}{X, X^{\prime}, A \rightarrow B \vdash Y, Y^{\prime}}\langle\rightarrow L\rangle
$$

That was no different to the Gentzen natural deduction elimination rule for the conditional, except for the slight modification allowing for multiple conclusions in a proof. (Here $\pi_{1}$ can have alternate conclusions $Y$, and this does not occur in the Gentzen-style natural deduction system. The circuit notation allows for a smoother representation for multiple conclusions.)

The sort of explanation works for the $\langle\rightarrow I\rangle$ node, but it works rather differently, in that the node is composed with a single preexisting proof: $\pi$ 
from $X, A$ to $B, Y$. Now the $\langle\rightarrow I\rangle$ node is plugged in to that proof, with the input port $B$ supplied by the $B$ exiting the proof $\pi_{1}$ (just as with the traditional $\langle\rightarrow I\rangle$ Gentzen rule, a proof ending in $B$ is extended by $A \rightarrow B$ ), but instead of selecting a number of premises to be discharged (which is a kind of 'action at a distance'), the connection betwen the single 'discharged' premise and the rule instance is made absolutely explicit by connecting the output $A$ port on the $\langle\rightarrow I\rangle$ node to the input $A$ port on the proof $\pi$. The remaining output port of the $\langle\rightarrow I\rangle$ node is marked with a dot, to indicate that it is the active port of this rule.

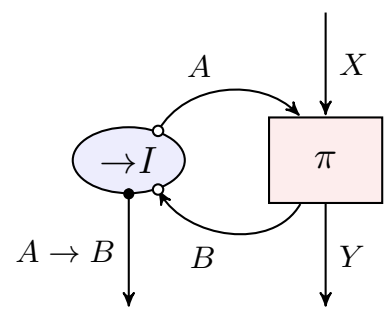

$$
\frac{X, A \vdash B, Y}{X \vdash A \rightarrow B, Y}\langle\rightarrow R\rangle
$$

Note that with both the $\langle\rightarrow I\rangle$ and $\langle\rightarrow E\rangle$ constructions, the active port on the connective node is on the periphery of the proof. Its wire is not linked to another part of the proof, but to the outside world. This means that the proof that is constructed is normal. A redudant $\langle\rightarrow I\rangle /\langle\rightarrow E\rangle$ pair is found when an ouptut $A \rightarrow B$ wire is connected to an input $A \rightarrow B$ wire. Then that wire will have dots at both ends, and the result is an active wire, which can then be simplified in the usual manner of normalisation.

This is part of the definition of a directed proof net. The other part of the definition, if we are to model classical logic, is the treatment of structural rules. This is necessary, because the 'discharge' achieved in $\langle\rightarrow I\rangle$ steps affects only one formula at a time. To discharge more than one formula, we need to contract those many instances into one. This can be done in a straightforward way, by pairing wires up.

If we have a proof with two premises $A$ or two conclusions $A$, a contraction ties these together and presents them as one instance, to be justified by another premise, or to be used as a conclusion in reasoning, or to be cut with another formula, or to be used in any other of a number of ways in a proof. This makes totally explicit the way that contraction appears in a proof and it detaches it from reliance upon a connective rule (as in Gentzen's natural deduction system) or embeds it into the linear nature of proof (as in Jaśkowski's). 

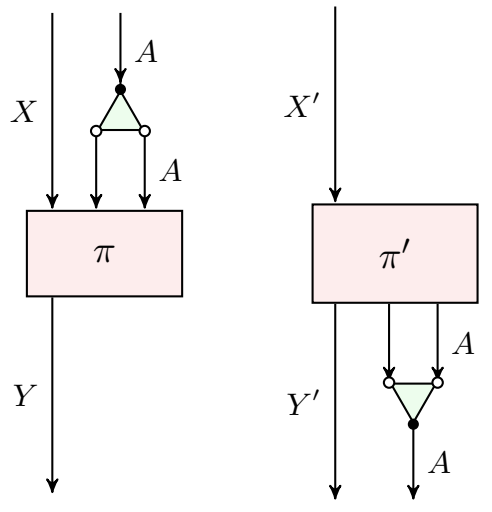

$$
\begin{aligned}
& \frac{X, A, A \vdash Y}{X, A \vdash Y}\langle W\rangle \\
& \frac{X^{\prime} \vdash A, A, Y^{\prime}}{X^{\prime} \vdash A, Y^{\prime}}\langle W\rangle
\end{aligned}
$$

Weakening is treated similarly. In a proof at any stage we can simply add another conclusion or another premise. Now, disconnected from any particular place in the proof itself.

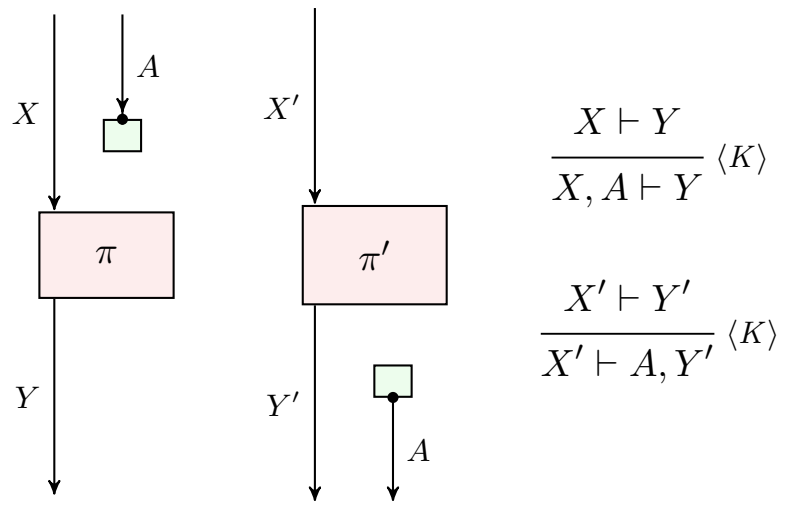

This, together with the $\langle C u t\rangle$ rule suffices to define the implicational fragment of classical logic. 


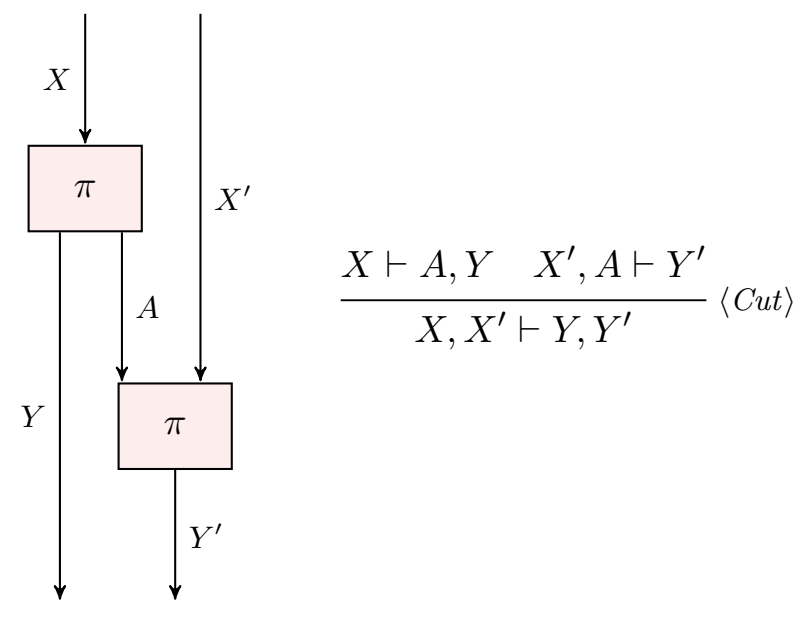

The resulting system is able to reproduce proofs corresponding nicely to sequent calculus proofs. Here is a natural deduction proof net corresponding to our derivation from $p \rightarrow r$ to $(p \rightarrow(r \rightarrow s)) \rightarrow(p \rightarrow(q \rightarrow s))$. Notice that the proof net has a very similar structure to our Gentzen proof on page 2, but the duplicate discharge of $p$ is marked by the contraction of the two $p$ wires into one by the contraction node, which is then supplied by the $\langle\rightarrow I\rangle$ node for $p \rightarrow(q \rightarrow s)$. The vacuous discharge of $q$ in the Gentzen proof is mirrored here by the point at which $q$ is weakened from the proof off the $\langle\rightarrow I\rangle$ node for $q \rightarrow s$. The rest is identical to the Gentzen proof, except for the radically different presentation as a circuit. 


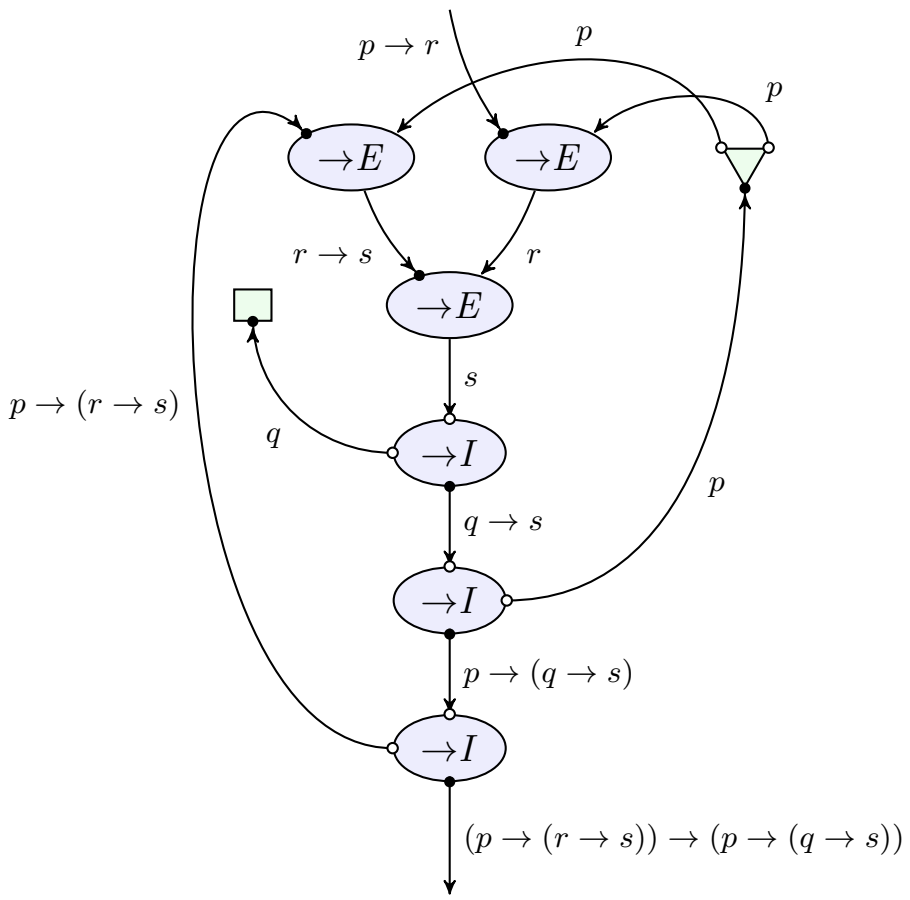

We can utilise the distinctive features of circuit notation by allowing for the downward branching of multiple conclusions in proofs. This allows for a simple, normal, directed proof net for Peirce's Law, satisfying the subformula property, corresponding to the usual sequent derivation:

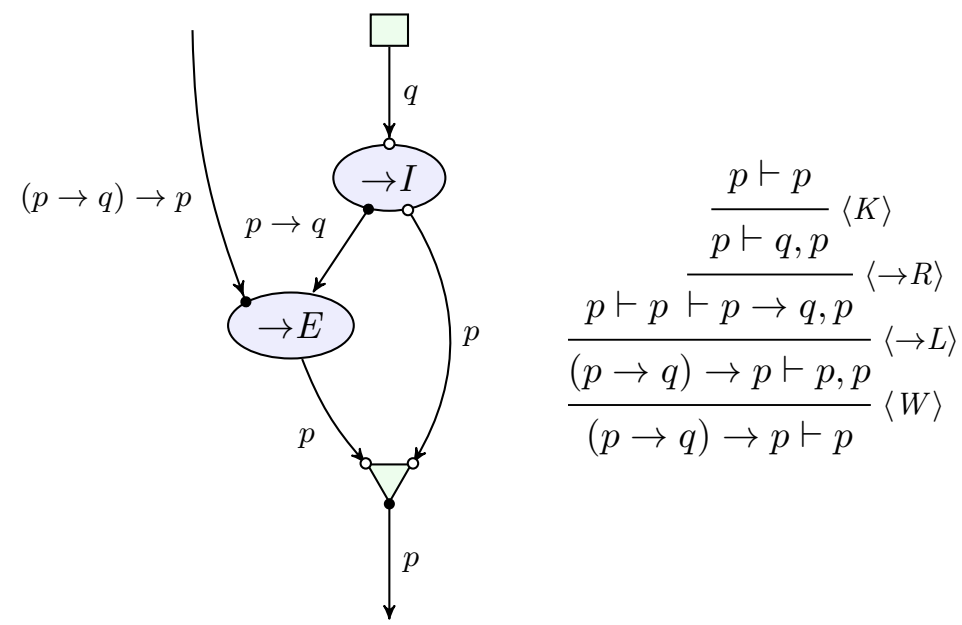


The result is a proof system for classical logic which has the same features as other natural deduction systems, but which adheres more closely to Gentzen's sequent calculus. As a result, it explicitly marks the presence or absence of structural rules, in the use of specific structural node types. This makes it more straightforward to include them or avoid them as applications demand, and it makes the features of structural rules independent of the operation of any particular connective type: the rules are modular in a way that Gentzen's or Jaśkowski's natural deduction systems are not. Cut-free derivations in the sequent calculus correspond neatly and precisely to normal directed proof nets.

Does this mean that directed proof nets are better than other natural deduction systems? Of course that is an impossible question to give a definitive answer. Better for what? It depends on what the aim of the exercise is. Jaśkowski and Gentzen style natural deduction systems have their own individual virtues. They are certainly more straightforward and more easy to learn than the circuit-notation used in directed proof nets. Single conclusion proofs are more accessible than mutiple conclusion proofs, too. But if the aim is to give an account of the kinds of inferential connections which are found in natural reasoning, then if classical sequent calculus derivations encode at least some of that reasoning, then it seems that proof circuit structures have their place. No other systems combine the virtues of separability of introduction and elimination rules for each connective (shared with Gentzen and Jaśkowski systems, but not with Hilbert systems), the modularity of take-it-or-leave-it options for structural rules, independent of any particular connective rules (shared with sequent calculi and their cousins, but not had by Gentzen or Jaśkowski), and the efficiency and parallelism of a natural deduction format (an advantage over merely working inside sequent calculi). For these reasons, directed proof nets deserve a place among the family of natural deduction proof systems.

Acknowledgements. Thanks to Allen Hazen, whose many conversations over the years on so many issues - not least, the subtle features of different presentations of natural deduction - have influenced me more than I can acknowledge in a footnote. Thank you, Allen, for patiently listening during innumerable occasions when I rambled on in front of whiteboards, for asking pertinent questions, and for guiding me through 100 years and more of the history of logic. My own research is much better for having worked with you. Thanks, too, to an anonymous referee for Studia Logica, whose comments helped to improve the content of the paper, as well as its clarity. 


\section{References}

[1] Anderson, A. R., and N. D. Belnap. Entailment: The Logic of Relevance and Necessity, volume 1. Princeton University Press, Princeton, 1975.

[2] Anderson, A. R., N. D. Belnap, and J. M. Dunn. Entailment: The Logic of Relevance and Necessity, volume 2. Princeton University Press, Princeton, 1992.

[3] Belnap, N. D., Display Logic. Journal of Philosophical Logic 11:375-417, 1982.

[4] Curry, H. B., Foundations of Mathematical Logic. Dover, 1977. Originally published in 1963.

[5] DošEn, K., The first axiomatization of relevant logic. Journal of Philosophical Logic 21(4):339-356, November 1992.

[6] Dunn J. M., and G. Restall. Relevance Logic. In D. M. Gabbay, (ed.), Handbook of Philosophical Logic, volume 6. Kluwer Academic Publishers, Second edition, 2002, pp. 1-136.

[7] Fitch, F. B., Symbolic Logic. Roland Press, New York, 1952.

[8] Gentzen, G., Untersuchungen über das logische Schliessen. Math. Zeitschrift 39, 1934.

[9] Gentzen, G., Die Widerspruchsfreiheit der reinen Zahlentheorie. Mathematische Annalen, 112:493-565, 1936.

[10] Girard, J.-Y., Linear Logic. Theoretical Computer Science 50:1-101, 1987.

[11] Girard, J.-Y., Y. LAfont, and P. TaYlor. Proofs and Types, volume 7 of Cambridge Tracts in Theoretical Computer Science. Cambridge University Press, 1989.

[12] JAśKowski, S., On the Rules of Suppositions in Formal Logic. Studia Logica 1, 1934. Reprinted in Polish Logic [17, pp. 232-256].

[13] Kneale, W., and M. Kneale. The Development of Logic. Oxford University Press, 1962.

[14] Kneale, W. C.. The Province of Logic. In H. D. Lewis, (ed.), Contemporary British Philosophy: Third Series, pages 237-261. George Allen and Unwin, 1956.

[15] Lemmon, E. J., Beginning Logic. Nelson, 1965.

[16] Mares, E. D. Relevant Logic: A Philosophical Interpretation. Cambridge University Press, 2004.

[17] McCall, S., (ed.). Polish Logic 1920-1939. Oxford University Press, 1967.

[18] Meyer, R. K., R. Routley, and J. M. Dunn. Curry's Paradox. Analysis 39:124$128,1979$.

[19] Negri, S., and J. von Plato. Structural Proof Theory. Cambridge University Press, Cambridge, 2001.

[20] O'Hearn, P., And D. Pym. The Logic of Bunched Implications. Bulletin of Symbolic Logic 5:215-244, 1999.

[21] Pelletier, F. J., A Brief History of Natural Deduction. History and Philosophy of Logic 20(1):1-31, March 1999.

[22] von Plato, J., Natural deduction with general elimination rules. Archive for Mathematical Logic, 40(541-567), 2001.

[23] Prawitz, D., Natural Deduction: A Proof Theoretical Study. Almqvist and Wiksell, Stockholm, 1965.

[24] Prior, A. N., Curry's Paradox and 3-Valued Logic. The Australasian Journal of 
Philosophy 33:177-182, 1955.

[25] Restall, G., An Introduction to Substructural Logics. Routledge, 2000.

[26] Restall, G., Multiple Conclusions. In P. Hájek, L. Valdés-Villanueva, and D. Westerståhl, (eds.), Logic, Methodology and Philosophy of Science: Proceedings of the Twelfth International Congress, pp. 189-205. KCL Publications, 2005. http://consequently.org/writing/multipleconclusions.

[27] Restall, G., Proofnets for s5: sequents and circuits for modal logic. In C. Dimitracopoulos, L. Newelski, and D. Normann, (eds.), Logic Colloquium 2005, number 28 in Lecture Notes in Logic. Cambridge University Press, 2007. http://consequently . org/writing/s5nets/.

[28] Robinson, E., Proof Nets for Classical Logic. Journal of Logic and Computation 13(5):777-797, 2003.

[29] Shoesmith, D. J., and T. J. Smiley. Multiple Conclusion Logic. Cambridge University Press, Cambridge, 1978.

[30] Slaney, J. K., Vagueness Revisited. Technical Report TR-ARP-15/88, Automated Reasoning Project, Australian National University, 1988.

[31] Slaney, J. K., A General Logic. Australasian Journal of Philosophy 68:74-88, 1990.

[32] Suppes, P., Introduction to Logic. van Nostrand, Princeton, 1957.

Greg Restall

School of Historical and Philosophical Studies

The University of Melbourne

Parkville 3010, Australia

restall@unimelb.edu . au 


\section{University Library}

\section{- M M I N E R VA A gateway to Melbourne's research publications}

Minerva Access is the Institutional Repository of The University of Melbourne

Author/s:

Restall, G

Title:

Normal Proofs, Cut Free Derivations and Structural Rules

Date:

2014

Citation:

Restall, G. (2014). Normal Proofs, Cut Free Derivations and Structural Rules. Studia Logica, 102 (6), pp.1143-1166. https://doi.org/10.1007/s11225-014-9598-4.

Persistent Link:

http://hdl.handle.net/11343/282858 